Invest. Pens. Crit. (ISSN 1812-3864; eISSN 2644-4119)

Vol. 9, No. 2, Mayo - Agosto 2021. pp. 16 -24

DOI: https://doi.org/10.37387/ipc.v9i2.232

Artículo Científico

\title{
Generación y segregación de residuos sólidos domiciliarios durante la cuarentena por Covid-19 en Panamá, estudio de caso
}

\author{
Household solid waste generation and sorting during Covid-19 quarantine \\ in Panama, case study
}

Norvin Requena Sanchez ${ }^{1}$, Dalia Carbonel Ramos ${ }^{2 *}, \&$ Erick Vallester ${ }^{3}$

${ }^{1}$ Equipo Técnico de Residuos Sólidos, Facultad de Ingeniería Ambienta, Universidad Nacional de Ingeniería, Perú. ${ }^{2}$ Equipo Técnico de Residuos Sólidos, Facultad de Ingeniería Ambienta, Universidad Nacional de Ingeniería, Perú.

${ }^{3}$ Universidad Tecnológica de Panamá UTP, Panamá.

* Autor por correspondencia: Dalia Carbonel Ramos, dcarbonelr@uni.pe Av. Túpac Amaru 210, Rímac, Apartado 1301, Lima, Perú.

Recibido: 30 de noviembre de 2020. Aceptado: 06 de abril de 2021

\section{Resumen}

La pandemia del Covid-19 ha provocado la declaratoria de cuarentenas en casi todos los países del mundo, en Panamá se mantuvo un confinamiento estricto entre los meses de marzo y setiembre. A pesar de la importancia de la gestión de los residuos sólidos en las ciudades la información sobre el impacto de la cuarentena en la generación y composición de residuos no ha sido lo suficientemente recopilada y difundida. En este estudio se presenta una metodología innovadora para recolectar información sobre los residuos domiciliarios sin salir de casa. Este trabajo muestra los resultados de una encuesta cerrada sobre hábitos de generación y segregación de residuos en seis provincias del país y los datos de caracterización y composición de los residuos sólidos domiciliarios en tres distritos de la provincia de Panamá. Los resultados de las encuestas evidencian las deficiencias del servicio de recolección de residuos en diferentes zonas del país. La generación per cápita domiciliaria de la provincia de Panamá es 0.409 kg/hab./día (27\% de error y 0.23 de desviación estándar), este valor es menor a otros hallados previamente lo que podría deberse a una disminución en la capacidad de compra de las familias ocasionada por el desempleo a raíz de la cuarentena. En la composición de residuos la proporción de residuos orgánicos y peligrosos aumentó. Los residuos orgánicos pueden haber aumentado por una mayor frecuencia y cantidad de alimentos preparados en casa. El aumento de residuos peligrosos es un indicador importante para emitir recomendaciones que reduzcan el riesgo del personal de servicio de recolección de residuos.

Palabras clave: Generación de residuos, Composición de residuos, Residuos sólidos domiciliarios, Covid19, Panamá. 


\begin{abstract}
The Covid-19 pandemic has caused quarantines in almost all the world countries. In Panama, strict confinement was maintained between March and September. Despite the importance of solid waste management in cities, information on the impact of quarantine on the generation and composition of waste has not been sufficiently collected and disseminated. This study presents a novel methodology to collect information on household waste without leaving the house. This work shows the results of a survey on waste generation and segregation habits in six provinces of the country; and data on household waste characterization and composition in three districts in the province of Panama. The survey results show the deficiencies of the waste collection service in different areas of the country. The per capita household generation in Panama's province is $0.409 \mathrm{~kg}$ per capita per day (27\% error and 0.23 standard deviation). This value is lower than others previously found, which could be due to a decrease in families' purchasing capacity caused by unemployment as a result of the quarantine. Regarding waste composition, the proportion of organic and hazardous waste increased. Organic waste may have increased due to a greater frequency and quantity of food prepared at home. The increase in hazardous waste is an essential indicator for issuing recommendations to reduce waste collection service personnel's risk.
\end{abstract}

Keywords: Waste generation, Waste composition, Household solid waste, Covid-19, Panama.

\title{
Introducción
}

El Covid-19 ha tenido un gran impacto en América Latina. Al momento de escribir este artículo en la región se presentan el 50\% de los casos totales en el mundo y el 55\% de fallecidos (WHO, 2020). De acuerdo a la Organización Mundial de la Salud los países con mayor número de casos en la región son Brasil, Argentina y Colombia (WHO, 2020). Los primeros casos de esta enfermedad se reportaron en la región el primer trimestre del 2020, a medida que los casos iban en aumento, en la primera quincena de marzo, la mayoría de países decretó cuarentena en diferentes modalidades: focalizada, obligatoria o recomendada (Chequea, 2020). Gran parte de las ciudades de la región mantuvo un confinamiento estricto hasta el mes de setiembre cuando estas condiciones fueron levantándose poco a poco (France24, 2020).

En Panamá el primer caso confirmado de Covid-19 se registró el 9 de marzo. El 13 de marzo se declaró emergencia nacional, en los días subsiguientes se cerraron las fronteras, se suspendieron las actividades recreativas, se estableció el toque de queda y se ordenó el cierre temporal de establecimientos comerciales y empresas (Minsa, 2020a). Estas condiciones estrictas de cuarentena se mantuvieron durante seis meses, a partir del 7 de setiembre se inició una reapertura gradual de las actividades económicas y desde el 14 de setiembre se eliminaron las restricciones de movilidad ciudadana. A la fecha en Panamá se han registrado 117,300 casos confirmados y 2,448 defunciones (Minsa, 2020b).

Gestión de residuos en América Latina y Panamá

En América Latina el 50\% de los residuos no se reciclan, la ONU calcula que 145.000 t/día se destinan a basurales, quema u otras prácticas inadecuadas; esto equivale al 27\% de la población regional, o los residuos generados por 170 millones de personas (Savino, Solórzano, Quispe, \& Correal, 2018).

La gestión de residuos sólidos en Panamá presenta deficiencias en todas sus fases (DefensoríaDelPueblo, 2007; Diéguez Pinto, 2018). Solo el 43.1\% de los municipios del país cuenta con un plan de manejo de residuos sólidos (Grau et al., 2015). En el Plan Nacional de Gestión Integral de Residuos Sólidos de Panamá (Ineco, 2017) se estima que los residuos generados por un 74\% de la población terminan en rellenos sanitarios; sin embargo, estos datos contrastan con el diagnóstico de vertederos de la AAUD (Autoridad de Aseo Urbano y Domiciliario, (AAUD, 2015)) donde se constata que gran parte de los municipios realizan la disposición final en vertederos a cielo abierto. Esta misma autoridad reporta la existencia de seis rellenos sanitarios en todo el país (AAUD, 2019) para un total de 81 municipios, e incluso esta información es debatible pues en la práctica el único relleno sanitario es el de Cerro Patacón que en realidad es considerado como un vertedero controlado. Todos estos lugares de disposición final no son manejados adecuadamente y generan graves problemas ambientales y de salud (DefensoríaDelPueblo, 2007; Perea, 2019; Rivas, 2015). En temas de reciclaje queda un largo camino por recorrer, una encuesta realizada a los alcaldes de $89 \%$ de los municipios de Panamá indica 
que, de forma general, no se realiza separación de los residuos (Ineco, 2017).

\section{Cambios en la generación de residuos en la pandemia en Panamá}

En el distrito de Panamá, la AAUD informó que entre la última semana de marzo y la primera quincena de abril se registró un aumento del $25 \%$ en la recolección de residuos de los 26 corregimientos del distrito capital (AAUD, 2020a). En el mes de agosto, tras la reapertura de algunas actividades comerciales, se observó gran cantidad de mascarillas y guantes usados en diversos puntos (AAUD, 2020b). Además, se reportó que 100 trabajadores de aseo entre personal administrativo y de campo se contagiaron con el Covid-19 (AAUD, 2020b).

Durante la pandemia de Covid-19 no ha habido una suficiente difusión sobre los datos de generación y reciclaje de residuos (Naughton, 2020). Esto es aún más evidente en el caso de América Latina donde la carencia de información sobre gestión de residuos sólidos de la mayor parte de los países de la región es un aspecto de fundamental importancia (Savino et al., 2018). En Panamá, por ejemplo, la información más reciente sobre la generación de residuos se encuentra en el Plan Nacional de Gestión Integral de Residuos Sólidos elaborado el 2017 (Ineco, 2017), la data a nivel distrital es escasa.

\section{Método}

\section{Objetivos}

El presente estudio presenta una metodología novedosa para recopilar información sobre la gestión de residuos sin necesidad de un trabajo de campo que aumente el riesgo de exposición. Es importante recolectar este tipo de datos para conocer cómo la pandemia y la cuarentena han impactado en la generación y composición de los residuos sólidos en las principales ciudades de la región, esta data será muy útil para la toma de decisiones en la gestión de residuos sólidos. En este estudio se presentan los resultados de hábitos de generación y segregación de residuos, así como datos de caracterización y composición de los residuos sólidos de la provincia de Panamá.

\section{Población, Muestra e Instrumento}

El estudio fue organizado por los alumnos del curso de "Gestión e ingeniería de residuos sólidos" de la Facultad de Ingeniería Ambiental de la Universidad Nacional de Ingeniería de Perú. La metodología del presente estudio ha sido previamente aplicada en algunas ciudades del Perú y Honduras. Con el objetivo principal de ampliar y difundir tanto la metodología como los alcances del estudio se contactó al docente y alumnos del curso de "Desechos sólidos" de la carrera de Saneamiento y Ambiente de la Facultad de Ingeniería Civil de la Universidad Tecnológica de Panamá para que participaran en el estudio. Este grupo fue la muestra seleccionada, las razones para su selección fueron el conocimiento en temas de gestión de residuos.

Los datos se recopilaron durante la primera semana de setiembre del 2020. El estudio consistió en una encuesta cerrada sobre hábitos de generación, número de personas en casa y segregación de residuos. En ella participaron 47 personas de las provincias de Panamá (30), Panamá Oeste (7), Colón (5), Herrera (2), Chiriquí (2) y Veraguas (1). El 91\% de los participantes viven en un área urbana.

Luego se hizo una capacitación en caracterización de residuos el 7 de setiembre, donde se enseñó a clasificar los tipos de residuos en orgánicos, inorgánicos aprovechables, no aprovechables y peligrosos. Así mismo en este taller se instruyó a los participantes a comenzar a elaborar un ecoladrillo, donde en una botella limpia y vacía colocarían residuos de envolturas de golosinas, bolsas de plástico, recibos de caja o cajeros, y otros similares. La segunda semana de setiembre los participantes ingresaron de manera virtual los pesos de los residuos previamente clasificados con lo cual posteriormente se obtuvo la cantidad y composición de residuos generados entre el 9 y el 15 de setiembre. En este estudio de caracterización participaron 17 personas de 3 distritos de la provincia de Panamá: Panamá (12), San Miguelito (3) y Chepo (2). 


\section{Procedimiento de análisis de datos}

Los resultados de la encuesta se analizaron porcentualmente. Una vez obtenido los pesos del grupo que participó, el procedimiento para el cálculo de la generación per cápita (GPC) y composición de residuos se calculó siguiendo el método recomendado por el Cepis (Centro Panamericano de Ingeniería Sanitaria y Ciencias del Ambiente) (Cantanhede, Sandoval Alvarado, Monge, \& Caycho Chumpitaz, 2005).

\section{Resultados}

\section{Indicadores de generación de residuos sólidos}

En la Tabla 1 se muestran los resultados de las preguntas hábitos de generación de residuos sólidos.

\begin{tabular}{|c|c|c|c|c|c|}
\hline \multicolumn{6}{|c|}{ Indicadores de generación de residuos sólidos } \\
\hline $\begin{array}{l}\text { ¿En qué tipo de } \\
\text { recipiente saca los } \\
\text { residuos sólidos de }\end{array}$ & $\begin{array}{l}\text { Bolsas } \\
\text { plásticas }\end{array}$ & $\begin{array}{l}\text { Bolsas de } \\
\text { papel }\end{array}$ & $\begin{array}{l}\text { Saco, costal } \\
\text { u otro } \\
\text { similar }\end{array}$ & $\begin{array}{l}\text { Caja de } \\
\text { cartón }\end{array}$ & Balde \\
\hline $\begin{array}{l}\text { casa para entregar al } \\
\text { camión recolector? }\end{array}$ & $75 \%$ & $5 \%$ & $8 \%$ & $10 \%$ & $3 \%$ \\
\hline $\begin{array}{l}\text { ¿Cada cuántos días } \\
\text { saca los residuos de }\end{array}$ & Diario & 2 días & 3 días & 4 días & $\begin{array}{l}\text { Más de } 5 \\
\text { días }\end{array}$ \\
\hline casa? & $21 \%$ & $29 \%$ & $19 \%$ & $11 \%$ & $21 \%$ \\
\hline
\end{tabular}

Tabla 1. Resultados de preguntas cerradas sobre hábitos de generación de residuos sólidos

Respecto al material usado para entregar los residuos al camión recolector el $75 \%$ emplea bolsas plásticas, $10 \%$ cajas de cartón, $8 \%$ sacos o costales y $5 \%$ bolsas de papel. El 21\% saca sus residuos a diario, $29 \%$ cada dos días, $19 \%$ cada tres días, $11 \%$ cada cuatro días y $3 \%$ cada cinco o más días.

\section{Percepción del servicio de recolección de residuos sólidos}

En la Tabla 2 se muestran los resultados de la pregunta relativa a la percepción del servicio de recolección de residuos sólidos.

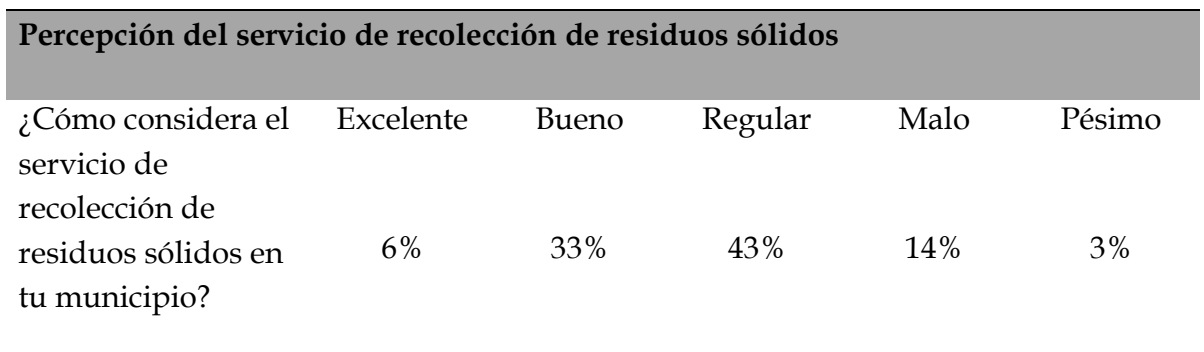

Tabla 2. Resultados de pregunta sobre percepción del servicio de recolección de residuos sólidos. 
Sobre la calidad del servicio de recolección brindado por el municipio $43 \%$ lo considera regular, $33 \%$ bueno, $14 \%$ malo, $6 \%$ excelente y $3 \%$ pésimo. Las sugerencias para la municipalidad se refieren a aumentar la frecuencia de recojo (20\%), definiendo días y cumpliendo con los horarios establecidos. Un respondiente del distrito Las Cumbres, provincia de Panamá, indicó que en ocasiones recolectan los residuos cada dos semanas. Se solicita también renovar la flota de camiones recolectores y evitar que se rieguen lixiviados en la pista; así mismo que se recojan los residuos que puedan caerse del camión recolector durante la ruta. Finalmente se sugiere implementar el recojo de residuos segregados o aumentar los puntos donde poder dejar residuos separados.

\section{Indicadores de segregación de residuos sólidos}

En la Figura 1 se presentan los resultados de las preguntas referentes a los hábitos de segregación de residuos sólidos.

El 56\% de los encuestados tiene capacitación o experiencia previa en segregación de residuos. El 57\% sabe qué es un ecoladrillo. Antes de la pandemia el 50\% de los respondientes entregaba los residuos inorgánicos generados en el domicilio a un reciclador (24\% algunas veces, $14 \%$ en muchas ocasiones y $13 \%$ siempre). El $40 \%$ no entregaba sus residuos a un reciclador y el 10\% manifestó que en su zona no hay recicladores. El 57\% no segrega los residuos que generan en el hogar; los participantes restantes separan sus residuos para reciclaje (14\%), para reciclaje y orgánico (14\%), para reciclaje y no aprovechable (8\%) y, sólo orgánico (6\%).

El 52\% reúsa sus residuos orgánicos en la elaboración de compost (48\%), como alimento para animales (17\%), preparación de humus $(5 \%)$ o productos de belleza $(2 \%)$.

El $40 \%$ separa y almacena el aceite usado en la cocina. Este aceite almacenado es botado junto con el resto de residuos (48\%), llevado puntos de acopio de aceite usado (36\%), y en dos casos empleado en casa como combustible o en la elaboración de jabón.

Finalmente, en relación al uso de bolsas reutilizables para las compras, $76 \%$ usan bolsas de tela siempre, $22 \%$ en muchas ocasiones y $2 \%$ algunas veces.

Figura 1. Resultados de preguntas sobre segregación de residuos sólidos
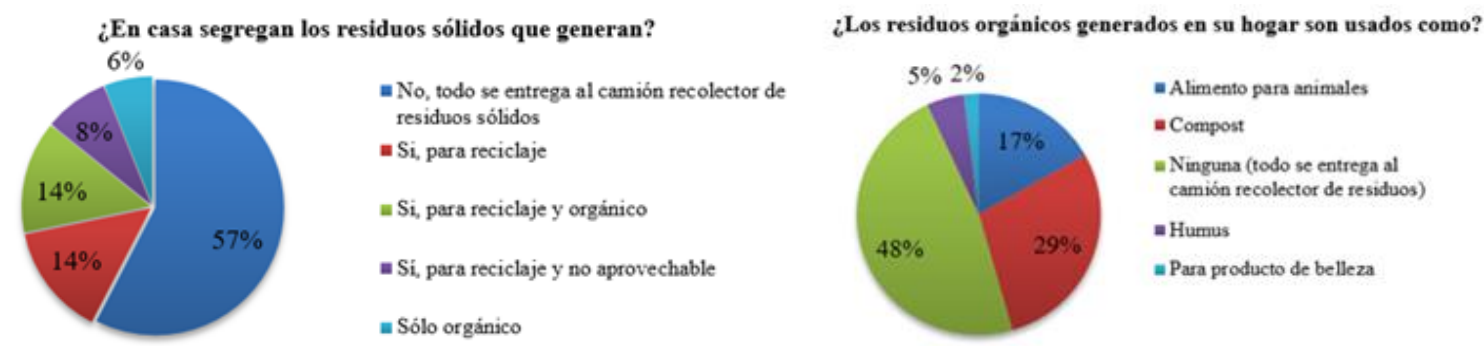

¿Antes de la pandemia entregaba los residuos sólidos inorgánicos generados en su domicilio a un reciclador?
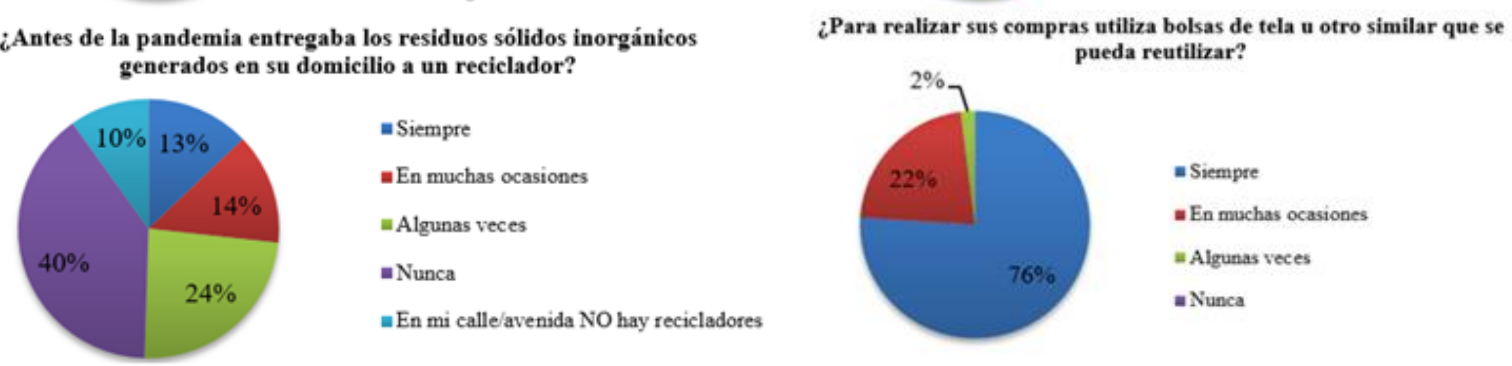


\section{GPC domiciliaria}

La GPC domiciliaria de la provincia de Panamá es 0.409 kg/hab./día, con una desviación estándar 0.23 y error del 27\%. En la Tabla 3 se presenta una comparación de la GPC domiciliaria de este estudio con otros valores definidos para el distrito de Panamá entre el 2002 y el 2015 y la GPC domiciliaria nacional del 2017.

\begin{tabular}{lcccc}
\hline Ámbito & Nacional & \multicolumn{2}{c}{ Distrito de Panamá } & Provincia de Panamá \\
\hline $\begin{array}{l}\text { Año } \\
\text { GPC }\end{array}$ & 2017 & 2002 & 2015 & 2020 \\
$\begin{array}{l}\text { domiciliaria } \\
(\mathrm{kg} / \text { hab./día })\end{array}$ & $0.66^{\mathrm{a}}$ & $0.590^{\mathrm{b}}$ & $0.591^{\mathrm{c}}$ & $0.409^{\mathrm{d}}$ \\
\hline
\end{tabular}

Tabla 3. Comparación de la GPC domiciliaria con valores del distrito de Panamá y nacional

a (Ineco, 2017) / b (JICA, 2003) / c Proyección para el 2015 (JICA, 2003) / d Este estudio

\section{Composición}

En la Tabla 4 se presenta la composición de los residuos durante la cuarentena en la provincia de Panamá.

\begin{tabular}{lc}
\hline Tipo de residuos & Porcentaje \\
\hline 1. RESIDUOS APROVECHABLES & \\
1.1 Residuos orgánicos & $43.99 \%$ \\
1.1.1 Residuos de preparación de alimentos y maleza & $31.47 \%$ \\
1.1.2 Sobras de guisos de la comida, aderezos y huesos & $12.52 \%$ \\
1.2 Residuos inorgánicos & $34.67 \%$ \\
1.2.1 Polietileno & $12.31 \%$ \\
1.2.2 Polietileno de alta densidad y polipropileno & $4.28 \%$ \\
1.2.3 Residuos aprovechables diferenciados & $10.14 \%$ \\
1.2.4 Ecoladrillo & $3.83 \%$ \\
1.2.5 Aceite usado & $4.11 \%$ \\
2. RESIDUOS NO APROVECHABLES & $21.34 \%$ \\
2.1 Residuos sanitarios & $12.54 \%$ \\
2.2 Residuos peligrosos & $4.07 \%$ \\
2.3 Inertes & $4.74 \%$ \\
\hline
\end{tabular}

Tabla 4. Composición de los residuos generados durante la cuarentena

Sumando los sub totales para cada categoría se tiene $43.99 \%$ de residuos orgánicos, $34.67 \%$ de residuos inorgánicos aprovechables y $21.34 \%$ de residuos no aprovechables. Las categorías con mayor proporción son los residuos de alimentos y malezas (31.47\%); residuos sanitarios (12.54\%); sobras de guisos y comidas $(12.52 \%)$; polietileno (12.31\%); y; residuos aprovechables diferenciados como papeles, empaques, vidrio o latas $(10.14 \%)$.

\section{Discusión}

\section{Percepción del servicio de recolección de residuos sólidos}

Como se refleja en las encuestas el servicio de recolección de los municipios en Panamá tiene muchos retos por delante, un diagnóstico hecho por la AAUD menciona que los municipios de las provincias de Panamá Oeste, Colón y Herrera presentan deficiencias en la recolección de los residuos (AAUD, 2015). En el distrito de Chame, provincia de Panamá Oeste, un funcionario de la municipalidad manifestó que la concesionaria "al inicio del servicio ... destinaba dos días de recolección a la semana, hoy en día se ve casi un día al mes" (AAUD, 2015). En el municipio de Chagres, provincia de Colón, no existe un sistema de recolección 
establecido de manera formal, por esta razón los pobladores queman sus residuos o los disponen directamente en el vertedero (AAUD, 2015). En la provincia de Panamá a excepción de los municipios de Panamá y San Miguelito, existen fuertes carencias en cuanto a personal y maquinaria para la recolección (AAUD, 2015).

\section{Indicadores de segregación de residuos sólidos}

La proporción de aceite que se dispone junto con el resto de residuos es preocupante pues en Panamá se estima que se generan alrededor de siete millones de galones de aceite al año, gran parte de los cuales termina en vertederos, ríos, quebradas y/ o en el suelo (DefensoríaDelPueblo, 2007).

\section{GPC domiciliaria}

En la Tabla 4 se observa que la GPC domiciliaria de la provincia de Panamá es menor a los valores nacional y distritales. En muchas ciudades la cuarentena ha ocasionado un aumento en la generación de residuos domiciliarios producto del mayor consumo de productos a domicilio y compras por internet (Pruett, 2020; Tenenbaum, 2020). Sin embargo, para un gran número de familias la cuarentena ha significado el cese de labores lo que ha llevado a una reducción de la capacidad adquisitiva del hogar (Ikiz, Maclaren, Alfred, \& Sivanesan, 2021; Naughton, 2020). Esta reducción puede explicar la menor cantidad de residuos generados.

\section{Composición}

En términos de composición la única fuente de comparación para el estudio de caso está en el Plan Nacional de Gestión Integral de Residuos (Ineco, 2017). Contrastando ambos resultados se observa que la proporción de residuos orgánicos es mayor en este estudio (34.67\% nacional y $43.99 \%$ en este estudio). Esto puede deberse a que al pasar mayor tiempo en casa haya aumentado la frecuencia y cantidad de preparación de alimentos lo que ha incrementado la cantidad de residuos orgánicos generados, así como las sobras de comida (Jribi, Ben Ismail, Doggui, \& Debbabi, 2020).

La proporción de plásticos disminuyó ligeramente (17.20\% nacional y $16.58 \%$ en este estudio). La proporción de residuos aprovechables diferenciados se redujo considerablemente $(32.50 \%$ nacional y $10.14 \%$ en este estudio). Este último resultado tiene relación con la disminución de la GPC, explicada por una menor capacidad de adquisición familiar, en las familias al parecer se están comprando una menor cantidad de productos empacados y procesados que usualmente tienen un mayor costo que los productos orgánicos (entendido como frutas, verduras y otros perecibles).

La cantidad de residuos peligrosos aumentó (1\% nacional y 4.07\% en este estudio). Para el estudio de caso los residuos peligrosos se componen en mayor proporción de mascarillas y guantes; en el caso de las primeras se generaron un total de dos unidades por persona durante una semana, un promedio de siete mascarillas a la semana por familia. Es importante tener en cuenta este dato debido al riesgo de biocontaminación que presentan los residuos como mascarillas y guantes. Estudios confirman que el coronavirus puede sobrevivir en superficies de plástico entre 50 a 100 horas (Kampf, Todt, Pfaender, \& Steinmann, 2020; van Doremalen et al., 2020). La Organización Panamericana de la Salud (OPS, 2020) ha emitido recomendaciones para la disposición de residuos que reduzcan el riesgo de contaminación por parte de los trabajadores del servicio de recolección de residuos. Aunque tangencialmente y de manera muy sucinta los Ministerios de Salud (Minsa, 2020c) y del Trabajo (MinisteriodeTrabajoyDesarrolloLaboral, 2020) de Panamá también han dado recomendaciones para la gestión de residuos durante la pandemia.

\section{Conclusiones}

La encuesta realizada a participantes de seis provincias de Panamá devela las deficiencias que presenta el servicio de recolección de residuos en diferentes zonas del país. Solamente la mitad de los encuestados segrega sus residuos y los entrega a un reciclador o los deposita en algún punto de acopio.

La GPC domiciliaria de la provincia de Panamá es 0.409 kg/hab./día, este valor representa una disminución respecto a valores hallados previamente a nivel nacional y para el distrito de Panamá. Dicha reducción puede deberse a una menor capacidad de adquisición familiar producto del desempleo ocasionado por la cuarentena.

En cuanto a la composición de residuos el mayor porcentaje (43.99\%) lo representan los residuos 
orgánicos, seguido de los residuos inorgánicos aprovechables (34.67\%) y por último los residuos no aprovechables (21.34\%). Comparando estos valores con los presentados el año 2017 en el Plan Nacional de Gestión Integral de residuos la proporción de residuos orgánicos y peligrosos aumentó, la de plásticos se mantuvo similar y la de residuos aprovechables disminuyó. Los residuos orgánicos pueden haber aumentado por una mayor frecuencia y cantidad de alimentos preparados en casa. El aumento de los residuos peligrosos es un tema al que debe de prestarse especial atención debido al riesgo que puede representar el manejo de residuos biocontaminados por parte del personal de servicio de recolección de residuos.

Cabe resaltar que el grupo seleccionado como muestra para la recopilación de datos son estudiantes universitarios familiarizados con el tema de gestión de residuos sólidos y temas ambientales, lo que ayuda a obtener mejores datos para el procesamiento.

\section{Referencias}

AAUD. (2015). Diagnóstico de los Vertederos a Nivel Nacional. Retrieved from http://www.aaud.gob.pa/Proyectos/Diagnostico/Diagnostico.pdf

AAUD. (2019). AAUD impulsa iniciativa para la gestión integral de residuos en áreas turísticas. Retrieved October 13, 2020, from Autoridad de Aseo Urbano y Domiciliario website: http://aaud.gob.pa/index.asp?sec=Noticias/2019\&id=09-06-2019

AAUD. (2020a). Autoridad de Aseo recoge 25\% más de basura en 18 días ante la actual situación de emergencia sanitaria. Retrieved October 12, 2020, from Autoridad de Aseo Urbano y Domiciliario website: https://sertv.gob.pa/autoridad-de-aseo-recoge-25-mas-de-basura-en-18-dias-ante-la-actualsituacion-de-emergencia-sanitaria/

AAUD. (2020b). Con la reapertura de otras actividades comerciales aumenta mascarillas y guantes tirados en las vías. Retrieved October 12, 2020, from Autoridad de Aseo Urbano y Domiciliario website: http://www.aaud.gob.pa/index.asp?sec=Noticias/2020\&id=08-25-2020

Cantanhede, A., Sandoval Alvarado, L., Monge, G., \& Caycho Chumpitaz, C. (2005). Hojas de divulgación técnica - CEPIS $N^{o}$ 97. Retrieved from https://sinia.minam.gob.pe/download/file/fid/39488

Chequea, L. (2020). ¿Qué medidas de aislamiento tomaron los países de América Latina durante la crisis por el coronavirus? Retrieved October 3, 2020, from Ojo Público website: https://ojopublico.com/1811/america-latina-se-encierra-e-impone-restricciones-por-la-pandemia

DefensoríaDelPueblo. (2007). Informe Especial sobre el Manejo de los Residuos Sólidos en Panamá. Retrieved from https://www.oceandocs.org/bitstream/handle/1834/8067/Manejo de Residuos Solidos en Panamá.pdf?sequence $=1$ \&isAllowed $=\mathrm{y}$

Diéguez Pinto, M. (2018). Manejo de desechos sólidos en la comunidad de Veracruz, Provincia de Panamá Oeste. Retrieved October 13, 2020, from Piragua - Fuego y agua website: https://piraguamdp.com/2018/03/02/manejo-de-desechos-solidos-en-la-comunidad-de-veracruzprovincia-de-panama-oeste/

France24. (2020). Termina la cuarentena obligatoria en varios países de Latinoamérica.

Grau, J., Terraza, H., Rodríguez, V., Diana, M., Rihm, A., \& Sturzenegger, G. (2015). Situación de la gestión de residuos sólidos en América Latina y el Caribe. Retrieved from https://publications.iadb.org/es/situacion-de-la-gestion-de-residuos-solidos-en-america-latina-y-elcaribe

Ikiz, E., Maclaren, V. W., Alfred, E., \& Sivanesan, S. (2021). Impact of COVID-19 on household waste flows, diversion and reuse: The case of multi-residential buildings in Toronto, Canada. Resources, Conservation and Recycling, 164, 105111. https://doi.org/10.1016/j.resconrec.2020.105111

Ineco. (2017). Plan Nacional de Gestión Integral de Residuos 2017 -2027. TOMO II. Retrieved from http://aaud.gob.pa/plangestion/Docs/PNGIR.pdf

JICA. (2003). Estudio sobre el plan de manejo de los desechos sólidos para la Municipalidad de Panamá en la República de Panamá. Ciudad de Panamá.

Jribi, S., Ben Ismail, H., Doggui, D., \& Debbabi, H. (2020). COVID-19 virus outbreak lockdown: What impacts on household food wastage? Environment, Development and Sustainability, 22(5), 3939-3955. https://doi.org/10.1007/s10668-020-00740-y

Kampf, G., Todt, D., Pfaender, S., \& Steinmann, E. (2020). Persistence of coronaviruses on inanimate 
surfaces and their inactivation with biocidal agents. Journal of Hospital Infection, 104(3), 246-251. https://doi.org/10.1016/j.jhin.2020.01.022

MinisteriodeTrabajoyDesarrolloLaboral. (2020). Protocolo para preservar la higiene y salud en el ámbito laboral para la prevención ante el Covid-19. Retrieved from https://www.mitradel.gob.pa/wpcontent/uploads/2020/03/Protocolo-Para-Preservar-La-Higiene-Y-Salud-En-El-Ambito-Laboral-ParaLa-Prevención-Ante-El-COVID-19.pdf

Minsa. (2020a). Bitácora del Evento Pandémico COVID-19, ler Cuatrimestre 2020. Retrieved from http://minsa.b-cdn.net/sites/default/files/publicacion-general/bitacora_-_junio_2020_0.pdf

Minsa. (2020b). COVID-19 07 de octubre de 2020. Retrieved from http://minsa.bcdn.net/sites/default/files/publicacion-general/informe_de_ejecucion_-_07102020.pdf

Minsa. (2020c). Lineamientos para el retorno a la normalidad de las empresas post Covid-19 en Panamá. Retrieved from https://mici.gob.pa/uploads/media_ficheros/2020/05/20/lineamientos-para-el-retorno-ala-normalidad-de-las-empresas-post-covid-19-en-panama-vc.pdf

Naughton, C. C. (2020). Will the COVID-19 pandemic change waste generation and composition?: The need for more real-time waste management data and systems thinking. Resources, Conservation and Recycling, 162, 105050. https://doi.org/10.1016/j.resconrec.2020.105050

OPS. (2020). Recomendaciones para la gestión de residuos sólidos, 11 de mayo del 2020. Retrieved from https://iris.paho.org/handle/10665.2/52108

Perea, C. (2019). Mi Ambiente multará a Urbalia por contaminación en vertedero de Cerro Patacón. Retrieved October 13, 2020, from Telemetro website: https://www.telemetro.com/nacionales/2019/11/25/miambiente-multara-urbalia-contaminacionvertedero/2301042.html

Pruett, M. (2020). Coronavirus Consumer Trends: Consumer Electronics, Pet Supplies, and More. Retrieved October 9, 2020, from Criteo website: https://www.criteo.com/blog/coronavirus-consumer-trends/

Rivas, F. (2015). La crisis de los residuos sólidos en Panamá. Retrieved October 13, 2020, from Apronad Panamá website: https://apronadpanama.wordpress.com/2009/10/27/la-crisis-de-los-residuos-solidosen-panama/

Savino, A., Solórzano, G., Quispe, C., \& Correal, M. C. (2018). Perspectiva de la Gestión de Residuos en América Latina $\quad y \quad$ el Caribe. Retrieved from https://www.unenvironment.org/es/resources/informe/perspectiva-de-la-gestion-de-residuos-enamerica-latina-y-el-caribe

Tenenbaum, L. (2020). The Amount Of Plastic Waste Is Surging Because Of The Coronavirus Pandemic. Retrieved October 9, 2020, from Forbes website: https://www.forbes.com/sites/lauratenenbaum/2020/04/25/plastic-waste-during-the-time-of-covid19/\#4e74328f7e 48

van Doremalen, N., Bushmaker, T., Morris, D. H., Holbrook, M. G., Gamble, A., Williamson, B. N., ... Munster, V. J. (2020). Aerosol and Surface Stability of SARS-CoV-2 as Compared with SARS-CoV-1. New England Journal of Medicine, 382(16), 1564-1567. https://doi.org/10.1056/NEJMc2004973

WHO. (2020). COVID-19 Weekly Epidemiological Update. Retrieved from https://www.who.int/publications/m/item/weekly-epidemiological-update---15-december-2020 